IV. Bühne und Leinwand 


\section{Das griechische Gesicht des Tanzes Eine Faszinationsgeschichte von Lady Hamilton zu Isadora Duncan}

Wer vom europäischen Tanz der letzten Jahrhunderte redet und dabei nicht den Volkstanz meint, denkt in der Regel an zweierlei Welten: an die romanisch-höfische Tradition des Balletts und an eine imaginierte griechische, die im Zeichen des Dionysos um die vorletzte Jahrhundertwende zum freien Ausdruckstanz geführt haben soll. Dass beide oder sogar alle drei Welten sich kreativ überschnitten haben, steht außer Frage. Dass es derartige Überschneidungen aber auch zwischen Bewegungskunst, Bildkunst, Theater und epischer Narration gab, ist erst seit der letzten Jahrtausendwende wirklich bewusst und Teil der Forschung geworden. Birgit Jooss und Ulrike Ittershagen legten fast zeitgleich umfangreiche Studien zur einer Kunstform vor, ${ }^{1}$ die unter dem Namen der `lebenden Bilder` sowie auch der >Attitudes` zurück in die Goethezeit führt, in der sie beliebt, aber nicht wirklich populär wurde. Heimisch wurde sie erst nach und mit der Erfindung des Stummfilms und der damit unerhörten Erweiterung der Bildfamilie. Als eine Art Hauskammertheater übte sie zunächst ein physiognomisches Regime eigener Art zwischen Kunstüberlieferung, Kunstausübung und Kunstvernichtung aus Vernichtung im Sinne von Grenzüberschreitung zum Boulevard einerseits, zur schieren Gymnastik andererseits.

Physiognomisch an alldem war zunächst vor allem die Position des Betrachters. Seit der antiken Deklaration der 'Physiognomik ' gab es zwischen Objekt und Betrachter das Sprachspiel der Deutung: Äußere Zeichen mussten und konnten als Zeichen einer inneren Verfasstheit erraten werden. Es waren die

1 Birgit Jooss: Lebende Bilder. Körperliche Nachahmung von Kunstwerken in der Goethezeit, Berlin 1999; Ulrike Ittershagen: Lady Hamiltons Attitüden, Mainz 1998. Jooss betont eindringlich den Unterschied zwischen >Attitüden Ittershagen gibt ihrer Darstellung eine eigene Note, indem sie mit einer derben Karikatur von Thomas Rowlandson einsetzt. Wirklich in Gang kam die Ideengeschichte wohl erst mit der großen Biografie von Kate Williams: England's Mistress: The Infamous Life of Emma Hamilton, London 2006, sowie der originellen Kunstgeschichte von Carrie J. Preston: Modernism's Mythic Pose. Gender, Genre, Solo Performance, New York 2011. 
lebensweltlichen Szenen des Sklavenmarktes oder des Pferdehandels, die ein Ratespiel zwischen Käufer und Verkäufer erzeugten, aber auch die Szene der Medizin, deren Semiotik zum Teil bis heute gilt. Nicht zuletzt galt seit dem Mittelalter das Wissen des Herrschers, welcher Phänotyp für den Dienst bei Hofe wohl wirklich geeignet sei, als physiognomisch. Ja, der Umgang bei Hofe überhaupt - man denke an Gracián - hielt sich an winzige Zeichen der Ratekunst. Als Lavater 1772 auf die Bühne dieses Diskurses trat, hatte die Physiognomik neben ihrer pragmatischen dann schon eine eindringliche Kunstgeschichte. Hier ging es nicht um Charakter, sondern um größtmögliche Lebendigkeit der zwar kunstvoll bearbeiteten, aber eben doch toten Materie. Winckelmann, der antike Skulpturen durch Ekphrasis »ins Leben hinein« betrachtete, mag als Erfinder eines pygmalionesken Schreibens gelten, wie später Mme de Staël in ihrem Buch über Deutschland hervorhob. ${ }^{2}$ Aber mit Lavater als Meister der Entzifferung von Körpern trat die Idee der Charade, der Zwang zum Erraten, als Modus eines "unsicheren Wissens" ins Blickfeld, und diesem Modus ist, musikalisch gesprochen, der folgende Gedankengang gewidmet.

Gerade wegen der vorauseilenden Modernität dieser Hermeneutik lohnt sich eine Darstellung im Rückblick, zumal unter Anleitung von Gabriele Brandstetter, der ersten Tanzwissenschaftlerin an der Freien Universität Berlin, seit 1996 im Amt, mit einem Akzent auf Kultur- und Literaturwissenschaft. In ihrem Buch über Körperbilder der Avantgarde (1995) $)^{3}$ fasste Brandstetter damals mit einem kühnen Wurf drei Initianten des Diskurses um 1900 zusammen: Aby Warburg, den Gründungsgeist der Kulturwissenschaft mit seinem Konzept der Pathosformeln von 1905, ${ }^{4}$ dann Isadora Duncan mit ihren Ideen zum Tanz der Zukunft von $1903^{5}$ und schließlich den Diagnostiker physiognomischen Schreibens, Hugo von Hofmannsthal, mit dem soge-

2 Helmut Pfotenhauer: Winckelmann und Heinse. Die Typen der Beschreibungskunst im 18. Jahrhundert oder die Geburt der neueren Kunstgeschichte, in: Beschreibungskunst Kunstbeschreibung. Ekphrasis von der Antike bis zur Gegenwart, hg. von Gottfried Boehm und Helmut Pfotenhauer, München 1995, S. 313-330.

3 Gabriele Brandstetter: Tanz-Lektüren. Körperbilder und Raumfiguren der Avantgarde, Frankfurt a.M. 1995. Soeben erschien die amerikanische Übersetzung: Poetics of Dance. Body, Image, and Space in the Historical Avantgardes, Oxford/New York 2015.

4 Vgl. Marcus Andrew Hurtig: Die entfesselte Antike. Aby Warburg und die Geburt der Pathosformel, Köln 2012.

5 Isadora Duncan: Der Tanz der Zukunft. Eine Vorlesung, Leipzig 1903. Im Folgenden zitiert nach der Neuausgabe von Karl Federn, Jena 1929. 
nannten Chandos-Brief von 1902, dieser berühmtesten Aufforderung zur Pantomime um 1900. ${ }^{6}$

Wie kam diese Kombination bei Brandstetter zustande? Tatsächlich konnte sie die Assoziation zwischen der amerikanischen Tänzerin und dem deutschjüdischen Kunsthistoriker einfach deshalb stiften, weil Duncan im Jahr 1899 die berühmte Primavera von Botticelli in Florenz besucht und damals an eben diesem Gemälde, dem Warburgs erstes Buch u.a. galt, offenbar tagelang einen ihrer frühesten Posen-Tänze einstudiert hatte.

Dass Warburg davon wusste, ist unwahrscheinlich; er hat sich ohnehin eher ungnädig über die Duncan geäußert. Vielleicht war ihm, dem Liebhaber der Renaissance, auch der demonstrative Hellenismus der Tänzerin unheimlich, denn Duncan tanzte 1903 nicht nur vor dem griechischen König Georg I. und dessen russischer Frau Olga, sie ließ sich mit ihrer Familie auch gleich einen ganzen Tempel unweit von Athen errichten. ${ }^{7}$ Was sie damals eigentlich bewog, die Szene des klassischen Balletts unter Protest zu verlassen, griechische Gewänder anzuziehen und sich schließlich zu einer Art physiognomischem Sprachrohr der Evolution zu machen, ist eine der Fragen, denen ich in der Folge nachgehen will; keineswegs als Erste, denn die Tanzwissenschaft ist in den letzten 20 Jahren geradezu stürmisch aufgestiegen. ${ }^{8}$ Aber kann man Tanz überhaupt als sphysiognomische Darstellung bezeichnen? Geht es dabei um Erkenntnisse durch die diaphane Körperbewegung hindurch auf charakterliche Befunde? Brandstetter spricht von Tanzlektüren, womit sie sowohl ihre eigene Hermeneutik bezeichnet als auch die Körperdarstellungswelt der Tänzer, die ja seit dem 17. Jahrhundert ihre Bewegungen im Raum auch choreografierten, also schriftlich notierten: griechisch choros graphein, den Tanz notieren. Notationen wie diese sind bis heute freilich nicht hermeneutisch physiognomisch gemeint, sondern vorschriftlich, präskriptiv. Und eben diese physiognomische > Vorschriftlichkeit umspielt immer wieder den Kern der hier vorgestellten Ideengeschichte. Duncans Tänze hätte man zwar wohl nie grafieren können, zu viel Improvisation herrschte bei ihr, wie bei so vielen anderen Solotänzern der Zeit. Aber ohne Vorlage tanzte auch sie nicht, und diese Vorlagen konnte man auch beschreiben, wie etwa Helene

6 Hugo v. Hofmannsthal: Ein Brief, in: Der Tag. Berlin, Nr. 489, 18. Oktober 1902 (Teil 1); Nr. 491, 19. Oktober 1902 (Teil 2).

7 Vgl. Isadora Duncan: My Life, London 1928.

8 Um nur auf die Aktivitäten der Freien Universität zu verweisen: Seit 2005 gibt es dort ein Zentrum für Bewegungsforschung unter Leitung von Gabriele Brandstetter, seit 2008 (zusammen mit Erika Fischer-Lichte und anderen) das vom Bundesministerium für Bildung und Forschung geförderte Research Centre of Interweaving Performance Cultures. 
von Nostitz, Freundin von Hofmannsthal. In ihren Erinnerungen von 1924 beschrieb sie einen Auftritt der Duncan um 1913:

Ich erlebte Isadora Duncan [...] kurz vor dem Kriege in Athen. Wie so oft, füllte sie einen ganzen Abend mit ihren Tanzphantasien aus. Nicht einen Augenblick ermüdete das Publikum. Denn hier war die Fülle des Lebens und der Kunst Ausdruck geworden. Sie tanzte die Säulen, die sie bei Sonnenuntergang und unter Sternen erlebt hatte. Sie tanzte die heiligen Schauer der Tempel, von denen der Weihrauch zu den Göttern aufsteigt, tanzte die Nymphen der Berge, Wälder und Flüsse, und die bacchantische Freude dionysischer Feste, tanzte die majestätische Tragik antiker Tragödien. Dann raste sie wieder daher wie eine Mänade und war die Furie, die Erinnye, die Orest in den Wahnsinn treibt, oder erschien als klagender Orpheus, als verlorene Seele, und tanzte die Sehnsucht, oder schritt als Iphigenie in weißem Gewande einher. Wie auf einer antiken Vase sank ihre Gestalt zu Boden und lag sinnend mit erhobenem Arm, und schwang sich dann wieder auf und war die freudige Göttin, mit Blumen bekränzt. ${ }^{9}$

Was für eine Textsorte haben wir hier vor uns - ein Stück Autobiografie, eine Apotheose, eine Ekphrasis eigener Art? Sicher jedenfalls eine bildungsbürgerliche Reminiszenz, denn es handelt sich um eine Paraphrase des ältesten griechischen Dialogs über den Tanz von Lukian, dem einflussreichsten Beispiel körperdeutenden Schreibens mit einer langen Geschichte. ${ }^{10}$ Offenbar war es schon bei den Römern üblich, Naturdarstellungen ebenso wie mythologische Geschichten in einer stummen Motorik nachzustellen, um sie alsdann erraten zu lassen.

9 Helene von Nostitz: Aus dem alten Europa, Leipzig 1924, Neuaufl. Frankfurt a.M. 1950, S. 161.

10 Vermutlich kannte oder besaß sie sogar die zeitgenössische Ausgabe Lukian. Sämtliche Werke, nach Wielands Übersetzung neu ediert von Hanns Floerke, in 5 Bänden, München/Leipzig 1911. Der Dialog Peri Orchesios - lat. De Saltatione - beschreibt in der alten Übersetzung von Pauly: »19. Nachdem ich aber der Indier und Aethiopier Erwähnung gethan, ist es nicht mehr als billig, auch ihrer Nachbarn, der Aegyptier, mit einem Worte zu gedenken; und so möchte ich denn behaupten, jene alte Fabel von dem ägyptischen Proteus spreche von nichts Anderem, als von einem geschickten Pantomimen, einem Meister in der Kunst der Nachahmung, der sich alle mögliche Stellungen und Figuren geben, und durch die Art seiner Bewegungen bald den weichen Fluß des Wassers, bald die Heftigkeit des flammenden Feuers, bald wieder den wilden Ungestüm eines Löwen, oder den Grimm eines Panthers, bald einen vom Winde bewegten Baum, kurz Alles darstellen konnte, was er nur immer wollte. Die Fabel aber, um das Wunderbare dieses Talentes zu erhöhen, erzählt diese Erscheinungen so, als ob er das Alles wirklich gewesen, was er durch Nachahmung darstellte. In der That steht dieselbe Kunst der Täuschung auch unsern heutigen Pantomimen zu Gebot: ist es doch oft, als ob sie sich in Einem Momente in ganz andere Wesen umwandelten, und den Proteus selbst zu spielen wüßten.« 
Greifen wir aber erst noch etwas aus. Die klassische Vorstellung von Physiognomik als einer Lehre der Gestaltdeutung bezieht sich ja auf stillgestellte Körper, genauer auf artefaktisch stillgestellte Phänotypen; für die bewegten hat man erst seit und mit Lichtenberg den Terminus 'Pathognomik eingeführt, als Deutungslehre vorrangig von Mimik, für die es aber noch kein Medium gab. Aber konnte man sie als bewegte beschreiben? Zu den Prämissen des Tanzdiskurses von heute gehört die Einsicht, dass sich der tanzende Körper weder so noch anders >deuten<, ja nicht einmal wirklich beschreiben lässt, eben weil es um nonverbale Künste geht. Gabriele Brandstetter hat aber, vielleicht intuitiv, schon vor ihrem Körperzeichenbuch ein kompaktes Lesebuch zum Beweis des Gegenteils ediert: Aufforderung zum Tanz (1993), ${ }^{11}$ ein literarisches Handbuch dieser Kulturtechnik, die natürlich von der Dichtung schon immer sehr eindrücklich evoziert wurde und deren elementare Bedeutung in den Sozialgefügen weltweit unbestritten ist. Vorschriftliche wie schriftliche Kulturen haben im Medium des Tanzes Motive aus existenziellen Praktiken wie Brautwerbung, Totenklage und Kriegsspiel inszeniert und als sozioästhetische topoi artikuliert. Dass man das diskursive Feld des Tanzes in Motive und Performanzen unterteilen muss, hat Peter Utz in seiner Studie zu Robert Walser überzeugend demonstriert und dabei kühn den Tanz als sprachliche Stilgebärde sui generis erwogen, die wiederum weniger als Ekphrasis denn als Dialog zwischen Autor und Leser gemeint sei. ${ }^{12}$ Der Leser muss etwa erraten, ob etwas ernst oder ironisch gemeint sei, ob er lachen soll oder nicht: Die Miene gerät in Tanzlaune.

Auch Helene von Nostitz' Beschreibung von Duncans Posen kann man als ekphrastisches Zitat bezeichnen; dass dessen Autorin selbst viel eher eine Meisterin des Dialogs war, hat Hofmannsthal in seiner Komödie Der Schwierige verherrlicht. Grundsätzlich aber evoziert sie mit ihrer Aufzählung von Duncan als Mänade, Orpheus, Orests Erinnye oder Iphigenie womöglich eben doch einfach Pathosformeln im Tanz - den Begriff hatte Aby Warburg zwar damals schon geprägt, aber nicht wirklich in Umlauf gebracht -, Pathosformeln also, die wir heute zu den spektakulärsten Erbschaften des Hellenismus zählen, dem sich die Tänzerin Isadora Duncan 1903 mit ganzer Seele ergeben hatte. »Weil die Posen der Griechen nur der Natur folgen, weil alle Götter der Griechen nur Naturgötter sind, nehme

11 Gabriele Brandstetter: Aufforderung zum Tanz, Stuttgart 1993.

12 Peter Utz: Der Schwerkraft spotten. Spuren von Motiv und Metapher des Tanzes im Werk Robert Walsers, in: Jahrbuch der Deutschen Schillergesellschaft 28 (1984), S. 384-404. 
ich konsequent Posen der griechischen Antike an, denn allein diese sind richtig auf unserer Erde. «13

Was immer innerhalb der Kulturwissenschaft aus Warburgs Pathosformeln geworden sein mag - deren ursprünglich zeitgenössische Tanz-Szene, vor allem die Idee der Pose, bot damals einen erstaunlich passenden Horizont für die Idee in sich geschlossener einfacher 'Mneme‘. Dieses Kunstwort sollte schon um 1900 als Pendant zum Wort 'Gener memorier- und vererbbare Einheiten beschreiben, ${ }^{14}$ kulturell resistent gegen Veränderungen, wie etwa Märchenmotive oder Archetypen, oder um 1900 die 'Elementargedanken von Adolf Bastian, Direktor des Berliner Völkerkundemuseums, oder etwas später die literarischen >Topoi< von Ernst Robert Curtius. Dass man auch visuelle Gesten und motorische Szenen von Körpern, gemalt oder gemeißelt, als `Mnemer analysieren und ähnlich wie Märchenmotive durch die Jahrhunderte und die unterschiedlichsten Medien verfolgen könnte und müsste, hat die 'Pathosformek jedenfalls zu einem der beliebtesten Werkzeuge der Kulturwissenschaft gemacht.

Die Tanzkultur selbst kennt dazu ein winziges motorisches und sinnfreies Äquivalent in den sogenannten Positionen des klassischen Balletts; seit dem 17. Jahrhundert gab es für Tänzer eine Reihe von festgelegten Haltungen des Körpers, vielleicht vergleichbar älteren Einteilungen auf dem Gebiet der Reiterei, deren Zweckdienlichkeit in der Kriegskunst noch unvergessen war. ${ }^{15}$ Neben diesen klassischen Posituren gab es aber seit dem 18. Jahrhundert auch die sogenannten 'Attitüden aus der Geschichte der Pantomime. Der griechische Satiriker Lukian hat die Pantomime, wie gesagt, schon um das Jahr 200 unserer Zeitrechnung in die Theoriegeschichte eingeschrieben. Sulzers Lexikon der schönen Künste erwähnt sie eher zurückhaltend, ${ }^{16}$ aber zur Goethezeit konstellierte sich dann eine lange Tradition der sogenannten 'lebenden Bilder als eigene Kunstgattung neben und mit den `Attitüden ${ }^{17}$ 'Topoi< wurden in diesem Kontext Figuren der antiken Erzähl- und Bildniskunst, Abbildungen auf Vasen, aber auch Statuen, insofern sie von lebenden Menschen nachgestellt wurden, nachgestellt eben, um vom Zuschauer erraten zu

13 Isadora Duncan: Der Tanz der Zukunft, S. 43.

14 Vgl. Richard Semon: Die Mneme als erhaltendes Prinzip im Wechsel des organischen Geschehens. Leipzig 1904.

15 Vgl. Ulrich Raulff: Das letzte Jahrhundert der Pferde. Geschichte einer Trennung, München 2015 .

16 Vgl. Johann Jakob Sulzer: Allgemeine Theorie der schönen Künste, Bd. 2, Leipzig 1774, S. 876-878.

17 Vgl. Birgit Jooss: Lebende Bilder, sowie Ulrike Ittershagen: Lady Hamiltons Attitüden. 
werden. Der Körper musste oder durfte spielerisch auf eine Bedeutung hin gelesen werden. Eine Narration, zumeist von Homer, steckte hinter jeder Pose; die Betrachter wurden zwangsläufig zu Physiognomisten, eben wie Helene von Nostitz, die später mit Lukian in der Hand die große Duncan entzifferte. Es war eine Art dialogischer Aneignung der Antike mit fließenden Übergängen zwischen Bild, Tanz, Literatur und Theater, zunächst ohne Musik, ohne inszenatorischen Aufwand, weshalb diese Kunst leicht auch in höfische wie bürgerliche Haushalte einziehen konnte. Lebende Bilder ebenso wie griechische Attitüden konnte man in jeder Hütte inszenieren - es bedurfte nur der Kenntnis des mythologischen Motivs, der antiken Kleidungsstücke, aber natürlich auch einer möglichst ausgebildeten gymnastischen Gelenkigkeit.

Beide Traditionen, die gymnastische wie die physiognomische, hatten um 1900 ihre Autoren neben Duncan und neben Hofmannsthal. Die gymnastische durchspielt etwa Frank Wedekinds Halbroman Mine-Haha, ${ }^{18}$ die seltsam verschlüsselte Geschichte eines hetärischen Internats nach antiken Vorbildern. Völlig im Zentrum aber steht sie im Klassiker der sogenannten physischen oder auch ästhetischen Gymnastik, der Bewegungslehre von Genevieve Stebbins, einer einflussreichen amerikanischen Tanzlehrerin. Stebbins' Buch ${ }^{19}$ erschien zuerst 1885 und in sechster Auflage 1902, ein Jahr vor Mine-Haha. Beide Werke repräsentieren eine hellenistisch inspirierte Tanzwelt, die gleich weit vom Volks- wie vom Gesellschaftstanz, vom klassischen Ballett wie vom Tanz der Avantgarde entfernt war. Es war eine Welt der Vorstufen zu alldem, eine Art motorische Propädeutik mit charakteristischer Dissonanz. Während Wedekind die jungen Mädchen in einem erotisch-schwülen spartanischen Tanzkollektiv aufwachsen und untergehen lässt, zielt Stebbins auf das Gegenteil: auf die Zurichtung weißer Amerikanerinnen aus unterschiedlichen sozialen Schichten, in der Absicht, sie für den Auftritt in höherer Gesellschaft oder auch für einen Auftritt auf der Bühne oder eben für beides zu trainieren, also eigentlich Gymnastik als Teil der Manierenlehre, ja Psychohygiene anzubieten. Das Buch von Stebbins hat dabei zunächst keine literarischen Absichten. Es ist ein präskriptiver Text über Körperbewegungen und -haltungen, der aber Lehrer und Schüler in eine ausdrücklich dialogische Szene versetzt und letztlich auf eine physiogno-

18 Frank Wedekind: Mine-Haha oder über die körperliche Erziehung der jungen Mädchen, München 1903.

19 Genevieve Stebbins: Delsarte System of Expression, 2. Aufl., New York 1887 [zuerst 1885]. 
mische Dressur hinausläuft. Das Projekt verdient eine etwas ausführlichere Betrachtung, denn Stebbins wird in Deutschland erst seit kurzem überhaupt von der Tanzforschung gewürdigt. ${ }^{20}$

Geboren in San Francisco 1857, gestorben 1934, kam Genevieve Stebbins als junges Mädchen zu einer Theaterausbildung bei Steele MacKaye, der seinerseits als Schüler des sagenumwobenen François Delsarte (1801-1871) dessen Lehre verbreitete. ${ }^{21}$ Delsarte gab seit 1835 Kurse für Sänger und Schauspieler, die mit der traditionellen Ausbildung gescheitert waren wie er selbst. ${ }^{22}$ Da er nicht schreiben konnte oder wollte, wie viele vergleichbar charismatische Lehrer, blieb dies seinen Schülern überlassen. Steele MacKaye ging nach dem Tod seines Lehrers 1871 zurück in die USA, wo er durch seine Frau den amerikanischen Philosophen Charles Sanders Peirce kennen lernte, der sich seinerseits für das triadische Zeichensystem von Delsarte interessierte. ${ }^{23}$

Delsartes Idee, wenn nicht Religion einer sphysischen Kultur - er war ein Anhänger von Saint-Simon - fasste Genevieve Stebbins im genannten Buch von 1885 zusammen. Von Tanz war darin nicht die Rede. Vielmehr wurde der sogenannte Delsartismus im letzten Drittel des 19. Jahrhunderts eine Art Ideologie des körperlichen Auftritts in den Vereinigten Staaten. Er verhieß Schönheit, Gewandtheit und moralische Makellosigkeit für Männer wie Frauen. Leitbild war die griechische Kalokagathie; schließlich hatte auch Stebbins einen hellenistischen Bildungshorizont, lange vor Duncan. 1881 besuchte sie auf einer großen Europareise die Museen in Paris, die auch Delsarte aufgesucht hatte, und prägte sich die Posen - also eben Pathosformeln avant la lettre - ein, die als sogenanntes statue posing schon in Delsartes Programm vorkamen.

Dem Historiker Taylor Lake zufolge erlaubte die soziale Praxis des statue posing schon um 1830 den amerikanischen Frauen der Mittelklasse, Theater im trauten Heim zu spielen und dabei zugleich klassische Bildung zu

20 So zuletzt energisch von Carrie J. Preston: Modernism's Mythic Pose.

21 Delsarte und Stebbins werden seit den 1980er Jahren Jahren von Nancy Lee Chalfa Ruyter in Erinnerung gehalten; vgl. Nancy Lee Chalfa Ruyter: The Cultivation of Body and Mind in Nineteenth-Century American Delsartism, London 1999.

22 Er verlor seine Stimme durch falsche Ausbildung, hielt aber am Plan einer Bühnenexistenz fest, wenn auch nur als Lehrer. Heute hält man ihn für einen Vorläufer von Stanislawski.

$23 \mathrm{Zu}$ Steele MacKaye vgl. Wendell Stone: Expressing Delsarte: Steele MacKaye's Contributions to the System of the French Master, in: Pioneering North America. Mediators of European Culture and Literature, hg. von Klaus Martens, Würzburg 2000, S. 215-224. 
extemporieren. ${ }^{24}$ Indem sie sich im pseudo-hellenischen Stil anzogen und Figuren und Themen aus der Kunstgeschichte nachstellten, konnten sie sich sozial höherstufen, weil - wie in Europa - auch die jungen Frauen der amerikanischen Oberschicht zwar pantomimisch agierten, aber sich gleichzeitig - anders als in Europa - gymnastisch dressieren ließen. Das statue posing war eine Weile lang in New York selbst in Museen und Theatern öffentlich Mode, bevor es im Boulevard verkam.

Noch im Todesjahr von Delsarte 1871 hellenisierte sich das Posieren dann aber wieder nachhaltig in Gestalt eines Musicals über Pygmalion and Galatea, komponiert von William Gilbert und hundertfach aufgeführt. ${ }^{25}$ Die Galatea spielte damals Mary Anderson aus der Delsarte-Schule, und die berühmte Szene, in welcher die Statue zum Leben erwacht, wurde jedes Mal mit größtem Beifall bedacht. Andersons Galatea gewann schließlich auch deshalb geradezu ikonische Geltung, weil sich die Schauspielerin mit weißer Perücke weiß schminkte, sogar weiße Arme und Schultern zeigte und so dem griechisch unterfütterten Idol einer weißen Amerikanerin huldigte. Winckelmanns Kunstlehre hatte hier ihren vielleicht politischsten Kontext gefunden und ihre Kernbotschaft: mittels Nachahmung der Antike diese Antike zurück ins Leben zu rufen.

Denn dieses 'Zurück ins Leben`, dieser Ausdruck der Wiedergeburt, war ja auch eine Formel mit ehrwürdiger europäischer Ideengeschichte. Seit 1772 gab es das Pygmalion-Melodram von Rousseau, inszeniert von Goethe in Weimar; und im gleichen Jahr konzipierte Goethe seinen Prometheus, der im gleichnamigen Gedicht wie auch im Drama Menschen schuf und Statuen beseelte. Und ebenfalls 1772 trat Lavater als physiognomischer Essayist auf den Plan, wenn auch noch anonym, der seinerseits Winckelmann huldigte und dessen Ekphrasis kunstvoller Leiber aus Stein. ${ }^{26}$

24 Vgl. Taylor Lake: The Delsarte Attitude on the Legitimate Stage: Mary Anderson's Galathea and the Trope of the Classical Body, in: Mime Journal 4/23 (= Essays on François Delsarte) (2004), S. 113-135.

25 William Gilbert (1836-1911), ein britischer Librettist für Operetten, die sogenannten sSavoy Operas‘, Begründer des Garrick Theatre, schrieb 1871 mit Pygmalion and Galatea sein bis dahin erfolgreichstes Stück.

26 Vgl. [Johann Kaspar Lavater]: Von der Physiognomik, Leipzig/Winterthur 1772. Sonderdruck eines Vortrags vor der Zürcher Naturforschenden Gesellschaft, anonym erschienen auf Anraten des Arztes und Schriftstellers Johann Georg Zimmermann. Vgl. zu diesem ganzen Komplex vor allem Inka Mülder-Bach: Im Zeichen Pygmalions. Das Modell der Statue und die Entdeckung der ’Darstellung« im 18. Jahrhundert, München 1998. 
Dieser Szene des Totstellens und Wiedererwachens, dieser 'Pygmalionformek, sit venia verbo, setzte nun Genevieve Stebbbins in ihrem Lehrbuch ein Denkmal. Sie prägte sie gleichsam als kleinste Münze dessen, was `Renaissance wörtlich meint. Mit folgendem Dialog setzt ihr Text ein:

A lovely day in spring. You are before me. Listen to my words: The first great step in the study of this art is the attainment of perfect flexibility. This is acquired by diligent practice of the decomposing exercises, as witness: I withdraw my willpower from fingers, the hand. Touch it. Do not shudder. Do you feel as if a dead thing had struck your living palm?27

Kaum zu glauben, dass die Vorstellung einer Leichenhand ihre Schülerinnen zum Mitmachen bewegen konnte. Aber es hat offenbar funktioniert. Die Lehrerin fährt fort: "Now I will show you the same phenomenon with forearm, entire arm, waist, spine, hips, knees, ankles, toes, jaw, eyelids. Now I fall.« ${ }^{28}$ Unwillkürlich wird man hier an eine Marionette denken, die von ihrem Spieler zur Ruhe gelegt wird, vor dem nächsten Auftritt. Und die Marionette gehorcht ${ }^{29}$ und zelebriert die Pygmalionformel. „Give me your hand and help me to rise", sagt die Lehrerin zur Schülerin in ihrem Lehrbuch. »I did not mean to startle you so. I have not even bruised myself. I simply withdrew my vital force into the reservoir at the base of the brain. The first great thing to be acquired is the flexibility of the joints." Denkwürdig modern mutet dieser Dialog heute an. Denn Stebbins gab naturkundliche Erklärungen für ihre Motorik, die gewiss erst mit Einbruch der darwinistischen Epoche formuliert werden konnten: "These exercises", fährt sie fort, "free the channels of expression, and the current of nervous force can thus rush through them as a stream of water rushes through a channel unclogged by obstacles. We name these exercises decomposing. «30

Leicht lassen sich in Stebbins' Vokabular die Vorlagen zu Isadora Duncans tänzerischem Credo erkennen. Stebbins evoziert vulgärvitalistisch eben das, was Duncan dann vollends naturalisiert und als Naturalisiertes wiederum gräzisiert: den freien Fluss der Bewegung, das Strömen des Wassers, die äußerste Beweglichkeit des Körpers und so fort. Zwar gehört die DelsarteSchule, wie gesagt, nicht wirklich in die Tanzgeschichte, aber alles, was die Duncan später am herrschenden Ballett, am Bühnentanz also, kritisierte, die beklemmende Zwangsschulung, die Enge der Kleidung und so weiter,

27 Genevieve Stebbins: Delsarte System of Expression, S. 11.

28 Ebd.

29 Vgl. ebd.

30 Ebd. 
all dies konnte sie hellenistisch inspiriert von ihren Lehrmeistern Delsarte und Stebbins kritisieren und abwerfen. Aber wohl kaum je ist der Aufruf zum Leben so nahtlos mit dem Bild des Totentanzes verknüpft worden wie im folgenden Satz:

"Sehen Sie denn nicht, dass unter den Röckchen und unter den Trikots deformierte Muskeln tanzen?«, fragte Duncan 1903 ihr Publikum. »Wenden Sie den Blick nicht $\mathrm{ab}$, denn unter den Muskeln sind die Knochen deformiert, ja ein deformiertes Skelett tanzt da vor Ihnen. ${ }^{31}$

Auf die Gesundung dieses Knochengerüstes legte die Delsarte-Gymnastik größten Wert - aber eben auch auf Bildung. Und diese reichte noch hinter die 1830er Jahre zurück. Denn was Isadora Duncan um 1900 in Florenz an der Primavera studierte, konnte sie in der Tat als Erbin einer rund hundertjährigen Tradition ins Werk setzen: lange vor aller dionysischen Anleitung, lange vor Nietzsche und vor Jane Harrison, der Lichtgestalt neuerer Klassikforschung.

Die leuchtende Figur hinter dieser ganzen griechischen Formgeschichte des statue posing war niemand anderes als Emma Hart, später Lady Hamilton und noch später Geliebte des Admirals Nelson. Geboren 1765 in einem Kohlennest in der Nähe von Liverpool, stieg sie zwischen 1786 und 1805 bis in die höchsten europäischen Kreise auf. Sie starb im Januar 1815 unter trostlosen Umständen in Calais, wohin sie vor ihren Steuerfahndern aus England geflohen war. ${ }^{32}$ Als Hart 1786 aus pikanten Gründen zu dem Kunstsammler Sir William Hamilton nach Neapel kam, hatte sie bei dem Maler bereits das Posieren in antiken Gewändern und Haltungen erlernt. ${ }^{33}$

31 Isadora Duncan: Tanz der Zukunft, S. 35. „Der Tanz war einst die erhabenste aller Künste - und soll es wieder werden. Aus der enormen Tiefe, in die er gefallen ist, wird er sich erheben müssen. Die Tänzerin der Zukunft soll den höchsten künstlerischen Grad erreichen und dadurch alle anderen Künste miterheben. Die Mission der Tänzerin ist das Moralische, Gesunde und Schöne in der Kunst zum Ausdruck zu bringen. All dem ist mein Leben gewidmet.« (ebd., S. 36). Eine deutlichere Absage an dionysische Konvulsionen lässt sich 1903 nicht denken!

32 Die ausführlichste Biografie stammt von Kate Williams: England's Mistress. Zum 200. Todestag hat die Casa di Goethe in Rom ihr einen opulentem Katalog gewidmet, nicht ohne die Häme der Männerwelt zu reproduzieren. Ausgerechnet George Grosz trat in die Fußstapfen von Thomas Rowlandson mit seinen Illustrationen zur Satire von Alfred Meyer, gen. Munke Punke: Lady Hamilton oder die Posen-Emma, Berlin 1923. - Birgit Jooss: Lebende Bilder, hat auf die Vorläufer der Attitudes aufmerksam gemacht.

33 Ihr eigentlicher Liebhaber Greville musste sie aus finanziellen Gründen an seinen reichen Onkel William Hamilton weitergeben; es gelang ihr, den viel älteren Mann zu heiraten und am Hof von Neapel eine eindrucksvolle Rolle zu spielen. 
Nun spielte ihr Leben plötzlich in einer gigantischen Sammlung von über tausend Vasen, Wandgemälden und Gläsern. Hamilton, damals britischer Gesandter am Hof von Neapel und neben seinem Interesse für Vulkanologie eben auch Kunstsammler, hatte 1772 ein Inventar erstellt und auch über die Ausgrabungen in Herculaneum berichtet. Darunter gab es eine Person mit geschlossenen Augen und deutlich erkennbarem Gesicht: beinahe unversehrt also seit dem schicksalhaften Vesuvausbruch im Jahr 79 n.Chr. Hamilton stellte sich seine Wiederbelebung vor als eine dramatische Auferstehung des Fleisches. Und um eine solche Auferstehung ging es dann eben auch in der Kunst der Emma Hart. Die Erfindung der >Attitudes`, die man gern ihr ebenso wie Sir William zuschreibt, bestand in der Reinszenierung von antiken Darstellungen nach gegebenen Vorlagen, und zwar vielen Vorlagen, die nacheinander gezeigt wurden, in einer anspruchsvollen Charade.

Was beim Maler Romney noch rein praktische Gründe hatte, der Wechsel von Pose und Kostüm, wurde nun in Neapel zu einer eigenen Choreografie, zu einer Art bewegtem Bild, einem Videomorphing avant la lettre. Die 'Attitudes` wurden im Saal des großen Palazzo auf ebener Erde gezeigt; Emma trug ein griechisches Gewand, und den Übergang von der einen zur nächsten Gestalt bewerkstelligte sie mit wenigen Handgriffen an ihren sechs Kaschmirschals und wenigen Requisiten. So nahe konnte das Publikum ihr kommen, dass man auch minimale mimische Bewegungen erkennen konnte. Die berühmteste Beschreibung dieser Kunst stammt von Goethe. Auf seiner italienischen Reise wurde er im März 1787 zum Augenzeugen - und mit der Druckfassung 1817 wurde sein Bericht kanonisch:

\section{Caserta, den 16. März 1787}

Wenn man in Rom gern studieren mag, so will man hier nur leben; man vergisst sich und die Welt, und für mich ist es eine wunderliche Empfindung, nur mit genießenden Menschen umzugehen. Der Ritter Hamilton, der noch immer als englischer Gesandter hier lebt, hat nun, nach so langer Kunstliebhaberei, nach so langem Naturstudium, den Gipfel aller Natur- und Kunstfreude in einem schönen Mädchen gefunden. Er hat sie bei sich, eine Engländerin von etwa zwanzig Jahren. Sie ist sehr schön und wohl gebaut. Er hat ihr ein griechisch Gewand machen lassen, das sie trefflich kleidet, dazu löst sie ihre Haare auf, nimmt ein paar Schals und macht eine Abwechslung von Stellungen, Gebärden, Mienen, usw., dass man zuletzt wirklich meint, man träume. Man schaut, was so viele tausend Künstler gerne geleistet hätten, hier ganz fertig in Bewegung und überraschender Abwechslung. Stehend, kniend, sitzend, liegend, ernst, traurig, neckisch, ausschweifend, bußfertig, lockend, drohend, ängstlich, usw., eins folgt aufs andere und aus dem andern. Sie weiß zu jedem Ausdruck die Falten des Schleiers zu wählen, zu wechseln, und macht sich hundert Arten von Kopfputz 
mit denselben Tüchern. Der alte Ritter hält das Licht dazu und hat mit ganzer Seele sich diesem Gegenstand ergeben. Er findet in ihr alle Antiken, alle schönen Profile der sizilianischen Münzen, ja den Belvederschen Apoll selbst. Soviel ist gewiss, der Spaß ist einzig! Wir haben ihn schon zwei Abende genossen. Heute früh malt sie Tischbein. ${ }^{34}$

Hamiltons Biografin berichtet, dass unmittelbar nach Goethes Besuch ein Strom von Reisenden in das Haus kam. "The Attitudes soon became crucial to any Self-respecting visitor's Grand Tour and essential to his descriptions of his encounters with classical culture. $\ll^{35}$

Mit diesen sogenannten >Attitudes entzückte Lady Hamilton zwischen 1785 und 1800 die gesamte europäische Gesellschaft, die sich in Neapel versammelt hatte. Angeblich beherrschte sie rund 200 Pantomimen, abgelauscht an meist griechischen Gemälden, Statuen und Vasenmalerei. Die Übergänge gestaltete sie offenbar mit wenigen Handgriffen so schnell, dass der Zuschauer von einer Stimmung in die andere katapultiert wurde - so wie es Goethe in seiner Italienischen Reise beschrieben hat.

Von Emma Hart-Hamilton gibt es unzählige Porträts, nicht nur vom Karikaturisten Thomas Rowlandson, sondern auch von den größten Porträtmalern der Zeit wie George Romney, von dem die Londoner National Portrait Gallery allein 26 Bildnisse besitzt, von Angelika Kauffmann und Elisabeth Vigée-Lebrun, von Tischbein und Friedrich Rehberg, dessen Vorlagen der italienische Künstler Pietro Antonio Novelli (1729-1804) nachgezeichnet hat (Abb. 1).

34 Johann Wolfgang Goethe: Werke. Hamburger Ausgabe, hg. von Erich Trunz, Bd. 11, 8. Aufl., Hamburg 1974, S. 209. Ergänzt hat Goethe diesen Bericht nach seinem zweiten Besuch am 27. Mai 1787: "Auffallend war mir ein aufrechtstehender, an der Vorderseite offener, inwendig schwarz angestrichener Kasten, von dem prächtigsten goldenen Rahmen eingefaßt. Der Raum groß genug, um eine stehende menschliche Figur aufzunehmen, und demgemäß erfuhren wir auch die Absicht. Der Kunst- und Mädchenfreund, nicht zufrieden, das schöne Gebilde als bewegliche Statue zu sehen, wollte sich auch an ihr als an einem bunten, unnachahmbaren Gemälde ergötzen, und so hatte sie manchmal innerhalb dieses goldenen Rahmens, auf schwarzem Grund vielfarbig gekleidet, die antiken Gemälde von Pompeji und selbst neuere Meisterwerke nachgeahmt. Diese Epoche schien vorüber zu sein, auch war der Apparat schwer zu transportieren und ins rechte Licht zu setzen; uns konnte also ein solches Schauspiel nicht zuteil werden« (ebd., S. 331).

Kate Williams: England's Mistress, Kindle edition, Position 2608. 


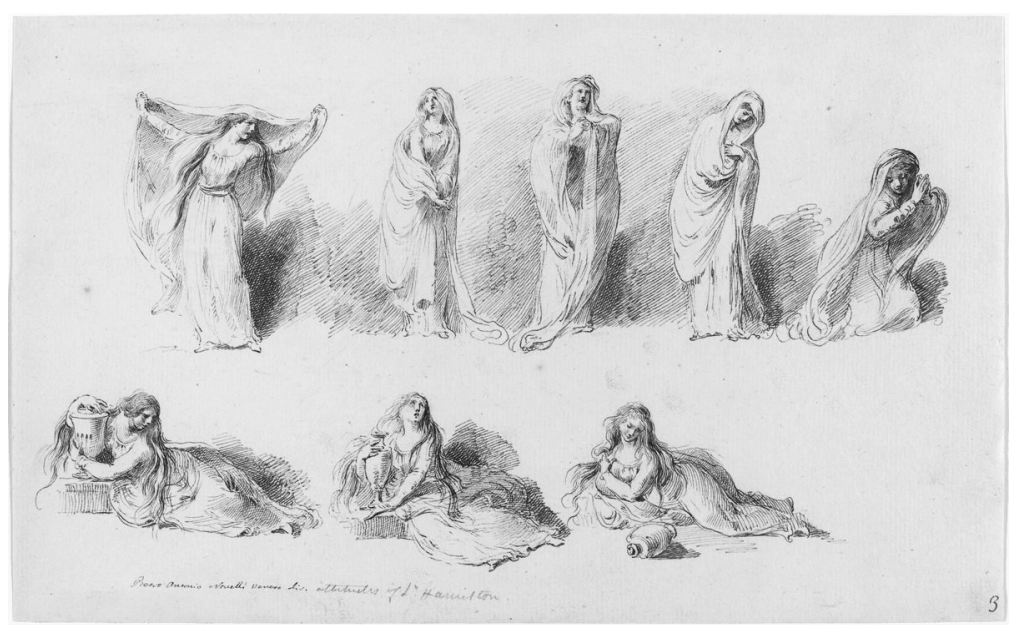

Abb. 1: Pietro Antonio Novelli nach Friedrich Rehberg, Lady Hamilton, Bleistift und braune Tinte, 1791, Washington, National Portrait Gallery,

Die Lady entzückte aber nicht nur mit ihrer Charade und mit den Bildungsgütern ihres Mannes und den berühmten ratefähigen Besuchern. Ihre Performance hatte als solche ein faszinierendes Janusgesicht. Wer sich um 1786 statt in Neapel in Berlin aufhielt, konnte dort Johann Jakob Engel begegnen, dessen Ideen zu einer Mimik von 1786 seine Schülerin Henriette Hendel-Schütz inspirierten und alsbald in die Fußstapfen Emma Harts treten ließen. HendelSchütz hatte Bilder von Hart-Hamilton gesehen - von Tischbein und Rehberg - und natürlich Berichte gehört. ${ }^{36}$ Und die Reihe der Nachahmerinnen und Nachfolgerinnen lässt sich problemlos verlängern. So entzückten in den 1790er Jahren die >Attitudes u u.a. auch die dänische Schriftstellerin Friederike Brun, selbst Augenzeugin in Neapel, die ihre Tochter Ida zur sittenreinen Nachfolgerin von Hamilton machen wollte. Etwas gespenstisch klingt die Begeisterung des fünfjährigen Kindes für Homer; ${ }^{37}$ aber in ihren Memoiren

36 Vgl. Johann Jakob Engel: Ideen zu einer Mimik, 2 Bde., Berlin 1785/86.

37 Friederike Brun: Wahrheit aus Morgenträumen und Idas ästhetische Entwicklung, Aarau 1824, S. 199: "Mit wahrem Heißhunger fielst du, als wir nun das neue große Haus in der Stadt bezogen, über meine aus Rom mitgebrachten Kupferwerke und übrigen Kunstsachen her. - Aber vor allem zog Dich Tischbeins Homer und sein großes Werk über die altgriechischen Gefäße an. Du zogest die edlen hohen Gestalten so zu sagen in dich herüber $[\ldots] . \ll$ 
von 1824 hat die Mutter auch jenes pathognomische Detail gespeichert, das schon Goethe auffiel: das Wunder der blitzschnell wechselnden Stimmungen und Mienen. Sie verwandelten diese >Attitudes eigentlich in ein Mienenspiel des ganzen Körpers oder umgekehrt den Körper in ein Gesicht - und damit in eine neue physiognomische Qualität. Statt Inhalte in eine bildungsbürgerliche Charade zu verschließen, offenbarte der mimische Anteil nun ein offensichtlich zutage tretendes Fühlen. ${ }^{38}$

Eben dieses Mienenspiel, oder besser Mienentheater, hat dann auch die ersten Performances des Delsarte-Lieblingsschülers Steele MacKaye beherrscht. Ein Theaterzettel von 1874 aus Boston (Abb. 2) zeigt die zirkusartige Demonstration der elaborierten Pathognomik von MacKaye, der die Schauspieltechnik seines französischen Tanzlehrers François Delsarte (1811-1871) mit großer Verve in den USA verbreitete.

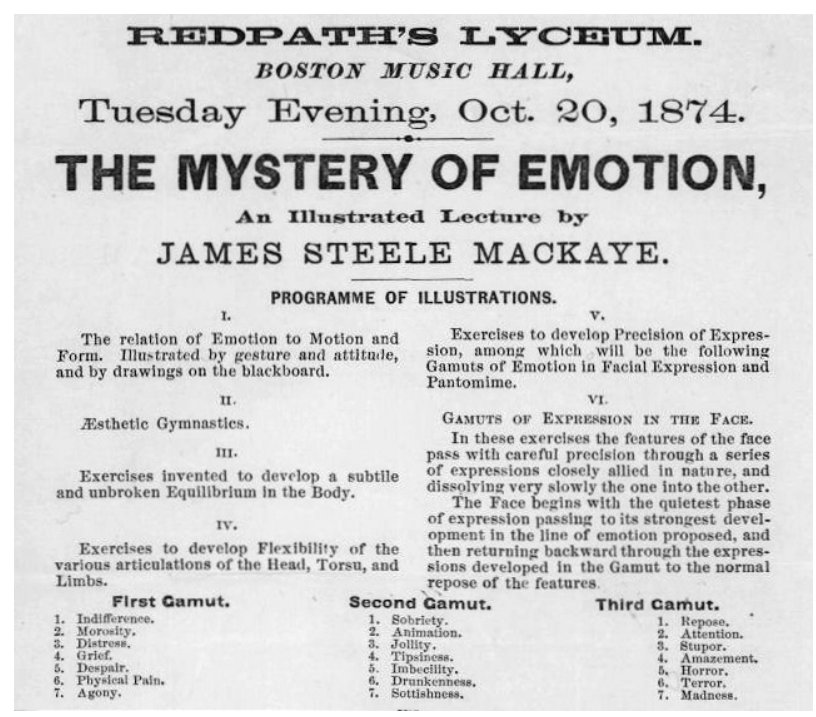

Abb. 2: Steeve MacKaye, Theaterzettel aus dem Boston Music Hall Archive

38 Susan Sontag hat das noch in ihrem Roman The Volcanolover (1993) evoziert. Die vielen Filme über das Leben der Emma Hart Hamilton seit 1921 konnten das natürlich nicht nachstellen. 
Unter dem Begriff des `Delsartismus` gelangte die 'gesunde` mimisch-expressive Technik des Selbstausdrucks so bis zum Ende des 19. Jahrhunderts in praktisch alle Haushalte der höheren Stände. Hamiltons und Delsartes Kunst der Stimmungswechsel tauchte bei ihm und später bei Stebbins unter dem Namen `Gamut of Expression` wieder auf.

Mit andern Worten: Mit und neben dem statue posing, das seine Zuschauer in eine Bildungscharade verwickelte, also ein Rätselraten in Gang setzte, entstand ein Mienenspieltheater mit der entgegengesetzten Botschaft: dem klaren und jedermann erkennbaren Ausdruck einer Emotion. Der Terminus >The Gamut of Expression ging von Delsarte in das Vokabular seiner Schule ein. Allerdings geriet dieses 'Gamut um 1870 sofort in den Sog des großen Buches von Charles Darwin: The Expression of the Emotions in Men and Animals, das 1872 erschien - und MacKaye reagierte, wie man sieht, blitzschnell. Womöglich kannte er, oder womöglich kannte sogar auch schon Delsarte die große Untersuchung des französischen Neurophysiologen Duchenne, dessen fotografisch gestützte Studien zum fazialen Ausdruck der Emotionen von 1862 Darwin seinerseits inspiriert hatten. ${ }^{39}$ Wie dem auch sei: Genevieve Stebbins hat sich dieser Seite des Delsartismus nicht angeschlossen. Ihre Ausführungen über das `Gamut of Expression`zeigen, dass sie vielmehr jeden Ausdruck in eine Narration zurückführte; und als ein solcher narrativer Ausdruck war er ja auch von Lady Hamilton in den Homer-Episoden und -Figuren evoziert worden. ${ }^{40}$

Auch Delsarte hatte Darwin nicht im Programm: Er starb ja 1871. Einer frühen sogenannten `Queerstudy< von Morris Meyer von 1994 kann man aber entnehmen, dass sich Delsarte um 1830, nach dem Bruch in seiner Karriere, möglicherweise ganz woanders, nämlich bei Balzac inspiriert hat. ${ }^{41}$ Dessen Traktate über das elegante Leben und über das Gehen von 1830 und 1833 liefern physiognomische Grundregeln - Balzac war bekanntlich ein großer Anhänger Lavaters. Im Horizont des Dandytums von Beau Brummel konzedierte er aber natürlich auch die Umkehrbarkeit der semiotischen Verhältnisse. Physische Zeichen und kulturelle Artefakte wie Wohnungen,

39 Vgl. Guillaume Benjamin Duchenne: Mécanisme de la physionomie humaine ou analyse électro-physiologique de l'expression des passions, 2ème édition, Paris 1876.

40 Etwa im 19. Kap. von Genevieve Stebbins: Aesthetic Gymnastics, New York 1893, S. 177: "You are standing idly in a room: a step on the stairs attracts your attention. The door opens to admit a person for whom you have an affection. You greet this person in delighted surprise."

41 Vgl. Morris Meyer: Under the Sign of Wilde. An Archeology of Posing, in: The Politics and Poetics of Camp, hg. von dems., London 1994, S. 65-93. 
Kleider, Kutschen, kurz, die gesamte Visibilität, mit Erving Goffman gesprochen, können erschlichen sein, können die Umwelt täuschen - aber auch auf das Subjekt zurückwirken, es sozusagen nach innen modellieren, wie etwa in Oscar Wildes Dorian Gray. ${ }^{42}$ Was in die ungeschriebene Schauspielkunde von François Delsarte also über Balzac einfloss, war umgekehrte Physiognomik - und was seine Schüler zu Papier brachten, war nicht physiognomisches Schreiben, sondern physiognomisches Vorschreiben: eine Didaktik der physischen Erscheinung, die Herstellung des Phänotyps, der vom Betrachter unmittelbar erraten werden sollte, wie jeder Schauspieler auf der Bühne. Es war eine schillernde Didaktik, die z.B. unter eugenischen Vorzeichen schließlich auch so etwas wie eine evolutionistische Diktatur errichten konnte. Die Botschaft, die Genevieve Stebbins schließlich in die Welt sandte, war jedenfalls im Grunde darwinistisch; "Semeiotics«, zitierte Stebbins angeblich Delsarte, »is the science of the organic signs by which aesthetics must study inherent fitness. ${ }^{43}$

Trotzdem: Die breitere Seite dieser Didaktik reicht tief in die Kunst, vor allem in die Pantomime der Stummfilmzeit, wie Carrie Preston und Ariane Martinez erst kürzlich nachgewiesen haben. Stumme Gebärden müssen zwar keine sinhärente Fitness beweisen, wohl aber müssen sie sich auf einen interkulturellen Code beziehen können, um international verstanden zu werden.

Entscheidender als die narrative Pantomime wurde um 1900 aber für den `Tanz der Zukunft ‘ die zunächst harmlos wirkende Formel von Genevieve Stebbins, wonach die gewünschte äußerste Flexibilität des Körpers nur durch vorherige restlose `Decomposition` zu erreichen sei, durch das `Sterben und wieder Auferstehen<, kurz: durch die Pygmalionformel. Eben dieser Formel hat sich die neben Duncan wohl berühmteste Tänzerin im Europa der Jahrhundertwende zugewandt: Loïe Fuller, das amerikanische Bewegungswunder, entzückte damals nicht nur die Besucher der Folies Bergère, sondern auch Dichter und Denker mit ihrem Flammentanz.

Vollkommene Beweglichkeit bei gleichzeitig vollständiger Zerstörung: Der berühmte sokratische, also wiederum hellenistische Dialog Eupalinos ou l'architecte von Paul Valéry versucht das 1921 in Worte zu fassen, die sein Freund Rilke dann an das deutsche Publikum adressierte:

42 Meyer macht auf die enge Bekanntschaft zwischen Oscar Wilde und Steele MacKaye aufmerksam und vertritt die plausible These, dass The Picture of Dorian Gray eine Station der Ideengeschichte des statue posing war.

43 Genevieve Stebbins: Delsarte System of Expression, S. 57. 
Socrates: Und Flamme, ist sie nicht auch die unfaßliche und stolze Gestalt der edelsten Zerstörung? - das, was nie wieder geschehen wird, geschieht prunkvoll vor unseren Augen! - das was nie wieder geschehen wird, muß notwendig mit dem größten Prunk geschehen, der sich denken läßt! - wie die Stimme blindlings singt, wie die Flamme singt, ganz außer sich, zwischen Stoff und Äther, und vom Stoff zum Äther grollend und wütend sich hinüberstürzt, - ist der große Tanz, o meine Freunde, nicht eigentlich die Befreiung unseres Körpers, der ganz besessen ist vom Geist der Lüge und von der Musik, die Lüge ist, und der sich trunken fühlt in der Verneinung der nichtigen Wirklichkeit [...]. ${ }^{44}$

Ob Valéry nun Genevieve Stebbins kannte oder nicht: Sein Text evoziert geradezu die `Decomposition` als Teil der Pygmalionformel, hier nun im ganz emphatischen genus sublime - mit der inzwischen grassierenden dionysischen Tanzidee verschränkt.

Das Ende des Dialogs ist bekannt. Die Flamme erlischt, die Tänzerin sinkt zu Boden - aber tot ist sie nicht, alsbald wird sie wieder entflammen. Goethes Selige Sehnsucht, Goethes Flammentod, Goethes orientalisches »Stirb und Werde« haben sich im hellenistischen Diskurs gehalten wie die Fliege im Bernstein.

44 Paul Valéry: Eupalinos oder über die Architektur, eingeleitet durch die Seele und der Tanz. Deutsch von Rainer Maria Rilke, Leipzig 1927, S. 112. 